\title{
Physiologic Pineal Region, Choroid Plexus, and Dural Calcifications in the First Decade of Life
}

\author{
M.T. Whitehead, C. Oh, A. Raju, and (D)A.F. Choudhri
}

\begin{abstract}
BACKGROUND AND PURPOSE: Calcifications of the pineal, habenula, choroid plexus, and dura are often physiologic. In the modern CT era with thin-section images and multiplanar reformats, intracranial calcifications have become more conspicuous. We aimed to discover the CT prevalence of pineal region, choroid plexus, and dural calcifications in the first decade of life.
\end{abstract}

MATERIALS AND METHODS: Five hundred head CTs from different patients (age range, 0-9 years) encountered during a consecutive 6-month period at a single academic children's hospital were reviewed retrospectively after excluding examinations with artifacts and pineal region masses/hemorrhage. All studies were performed on a 320-detector CT, with 0.5 -mm collimation and a $512 \times 512$ matrix. Five-millimeter reformatted axial, sagittal, and coronal images were analyzed for location and extent of intracranial calcifications.

RESULTS: The mean age was $3.5 \pm 5.7$ years (range, $0-9$ years). There were 285 males ( $57 \%$ ) and 215 females (43\%). Pineal calcifications were present in $5 \%$ ( $n=25$; age range, 3.2-8.9 years; median, 7 years). Habenular calcifications were found in $10 \%$ ( $n=50$; age range, $2.8-8.8$ years; median, 7 years). Twelve percent $(n=58)$ had choroid plexus calcifications, (age range, 0.1-8.8 years). Dural calcifications were rare, present in $1 \%$ ( $n=6$; age range, $2.9-8.7$ years).

CONCLUSIONS: Physiologic intracranial calcifications may be found in the first decade, principally in children older than 5 years. Most epithalamic calcifications are habenular. Pineal and habenular calcifications were never present in children younger than 3 and 2 years, respectively. Choroid plexus calcifications may be present in the very young. Dural calcifications are rare.

$\mathbf{P}$ hysiologic intracranial calcifications become increasingly prevalent with advancing age but can be found in children and adults. Pineal region calcification is known to be present histologically from fetal life to adulthood. ${ }^{1}$ Benign calcifications in the pineal gland, habenula, choroid plexus, and dura should not be mistaken for hemorrhage or pathologic tumoral or metabolic mineralization. Thus, it is important to know where physiologic intracranial calcification is expected, and the age when it becomes detectable on imaging.

The widely held dictum that pineal region calcifications found

Received July 17, 2014; accepted after revision September 5.

From the Departments of Radiology (M.T.W., C.O., A.R., A.F.C.) and Neurosurgery (A.F.C.), University of Tennessee Health Science Center, Memphis, Tennessee; Department of Radiology (M.T.W., A.R., A.F.C.), Le Bonheur Children's Hospital, Memphis, Tennessee; and Department of Radiology (M.T.W.), Children's National Medical Center, Washington, DC.

Paper previously presented at: American Society of Neuroradiology Annual Meeting and the Foundation of the ASNR Symposium; May 17-22, 2014; Montreal, Quebec, Canada.

Please address correspondence to Asim F. Choudhri, MD, Department of Radiology, Le Bonheur Children's Hospital, 848 Adams St-G216, Memphis, TN 38103;

e-mail: achoudhri@uthsc.edu

http://dx.doi.org/10.3174/ajnr.A4153 in those younger than 6 years of age are always pathologic no longer holds true in the modern CT era. ${ }^{2}$ Previous imaging studies that generated the currently established age thresholds examined intracranial calcifications on more primitive CT scanners with smaller matrices and thicker sections. Furthermore, sparse literature exists regarding the prevalence of dural and choroid plexus calcifications on head CTs in children. We sought to discover the prevalence of intracranial calcifications in the pineal gland, habenula, choroid plexus, and dura in the first decade of life by using contemporary CT technology.

\section{MATERIALS AND METHODS}

This Health Insurance Portability and Accountability Act-compliant retrospective study was performed after institutional review board approval. The imaging database at a single academic children's hospital was searched for all noncontrast head CTs from separate patients younger than 10 years of age obtained during a consecutive 6-month period. The search yielded 507 examinations. Patients with entities known to be associated with calcifications were excluded. Examinations with excessive motion artifacts, pineal region masses, or evaluation-limiting hemorrhage were also excluded. All examinations were performed on a 


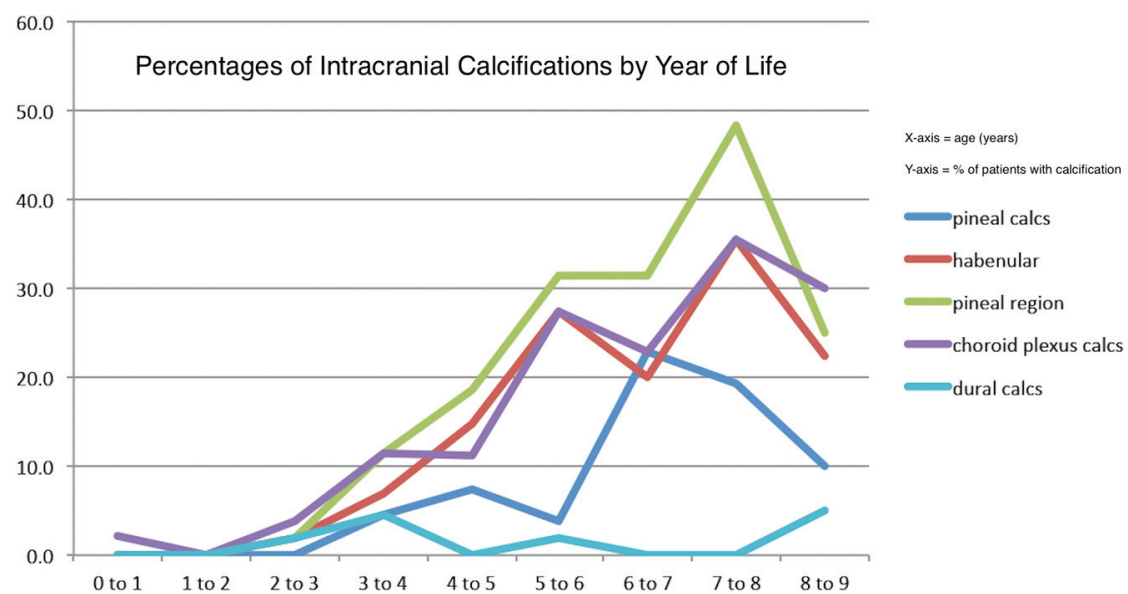

FIG 1. Chart depicting the percentage of patients with calcifications ( $y$-axis) in various regions by year of life (x-axis).

\begin{tabular}{lcc}
\multicolumn{3}{l}{ Location of specific intracranial calcifications } \\
\hline \multicolumn{1}{l}{ Location of } \\
Calcification & No. of Patients & Age Range (yr) \\
\hline Pineal gland & $25 / 500(5 \%)$ & $3.2-8.9$ (median, 6.7) \\
Habenula & $50 / 500(10 \%)$ & $2.8-8.8$ (median, 6.5) \\
Pineal region & $63 / 500(13 \%)$ & $2.8-8.9$ (median, 6.5) \\
Choroid plexus & $58 / 500(12 \%)$ & $0.1-8.8$ (median, 6.3) \\
Dural & $6 / 500(1 \%)$ & $2.9-8.7$ (median, 4.5) \\
\hline
\end{tabular}

320-detector CT scanner (Aquilion ONE; Toshiba Medical Systems, Tokyo, Japan) with $0.5-\mathrm{mm}$ collimation and a $512 \times 512$ matrix. The epithalamus, choroid plexus, and dura were analyzed for calcifications on 5-mm axial, coronal, and sagittal reformatted images in each case. Epithalamic calcifications included either pineal, habenular, or both. Epithalamic calcifications were also subdivided into pineal or habenular on the basis of their distinct location; focal pineal parenchymal or pineal wall calcifications were classified as "pineal," while pineal adjacent calcifications were considered "habenular." The latter are generally localizable anterolateral to the pineal gland along the medial thalamic margins.

Calcification positivity was defined as hyperattenating material respective to gray matter. In addition to group analysis, we evaluated patient subsets on the basis of age. We recorded the total number of patients in each year of life and calculated the percentages of pineal, habenular, choroid plexus, and dural calcifications in patients of each age (range, 0-9 years). Pineal calcifications were classified as punctate and/or single or larger and/or numerous $(>1)$. All encountered pineal cysts were recorded and manually measured by calipers on a PACS in the maximal diameter; mean pineal cyst sizes were then calculated. Dural and choroid plexus calcifications were grouped by location. Choroid plexus calcifications were classified as either punctate and/or few or large and/or multiple. The potential relationship between choroid plexus and pineal calcifications was explored.

Images were retrospectively reviewed in a blinded manner by 2 fellowship-trained neuroradiologists with American Board of Radiology subspecialty certificates in neuroradiology (M.T.W. and A.F.C.). Both readers had $>3$ years of clinical experience after board certification. A consensus reading was reached in all cases. Each patient's electronic medical record was researched for pre- senting symptomatology, demographic information, and pertinent physical examination findings.

Five hundred patients met the inclusion criteria, with a mean age of $3.5 \pm 5.7$ years (median, 2.5 years; range, 0.0-9 years). The percentages of patients with intracranial calcifications in each age group are shown in Fig 1. There were 285 males (57\%) and 215 females (43\%). The most common clinical indications for the examinations were trauma $(n=209)$, seizures $(n=66)$, shunt $(n=57)$, and headache $(n=41)$. None of the patients had undergone prior therapeutic radiation, and no histories of metabolic disorders, endocrinopathies, or renal diseases could cause calcium-phosphate imbalance and premature or excessive intracranial calcification. One patient had a history of tuberous sclerosis but had no intracranial calcification. None of the patients had documented histories of TORCH infection or neurofibromatosis type 1, entities known to be associated with calcifications in or around the choroid plexus. ${ }^{3}$

\section{Data Collection and Statistics}

Data were stored and analyzed in a spreadsheet (Excel 2011; Microsoft, Redmond, Washington) and in SPSS (Version 20.0; IBM, Armonk, New York). Discrete variables were evaluated by using the $\chi^{2}$ or Fisher exact test, as appropriate. Continuous variables were analyzed for significance by using the Kruskal-Wallis test with a nonparametric posttest. Correlations were made by using the Pearson product-moment correlation coefficient. $P$ values $<$ .05 were considered significant.

\section{RESULTS}

Age and intracranial calcification presence were positively correlated $(P<.0001)$ (Fig 1). Most $(97 \%)$ intracranial calcifications were found in patients older than 5 years. There was no significant difference in sex $(P=.41)$ or race $(P=.28)$ of patients with and without calcifications. A summary of location-specific intracranial calcifications is provided in the Table.

\section{Epithalamus}

Epithalamus (pineal and/or habenular) calcifications were present in 13\% (63/500). Pineal and habenular calcifications were positively correlated $(P<.0001)$. Pineal calcifications were present in $5 \%$ ( $n=25$; age range, $3.2-8.9$ years; median, 7 years). One percent $(6 / 500)$ of patients were younger than 6 years of age (Fig $2)$. Pineal calcifications were single and/or punctate in $71 \%$ (17/24) and larger and/or more numerous in 29\% (7/24; range, 7-9 years).

Pineal cysts were found in $3 \%(n=15$; age range, $0.7-7.5$ years; median. 2.6 years). These ranged from 4 to $15 \mathrm{~mm}$ (median, $5 \mathrm{~mm}$ ). Four of these 15 patients had epithalamic calcifications involving the habenula $(n=4)$ and/or pineal gland $(n=1)$.

Habenular calcifications were found in $10 \%(n=50$; age range, $2.8-8.8$ years; median, 7 years; Fig 2 ). 

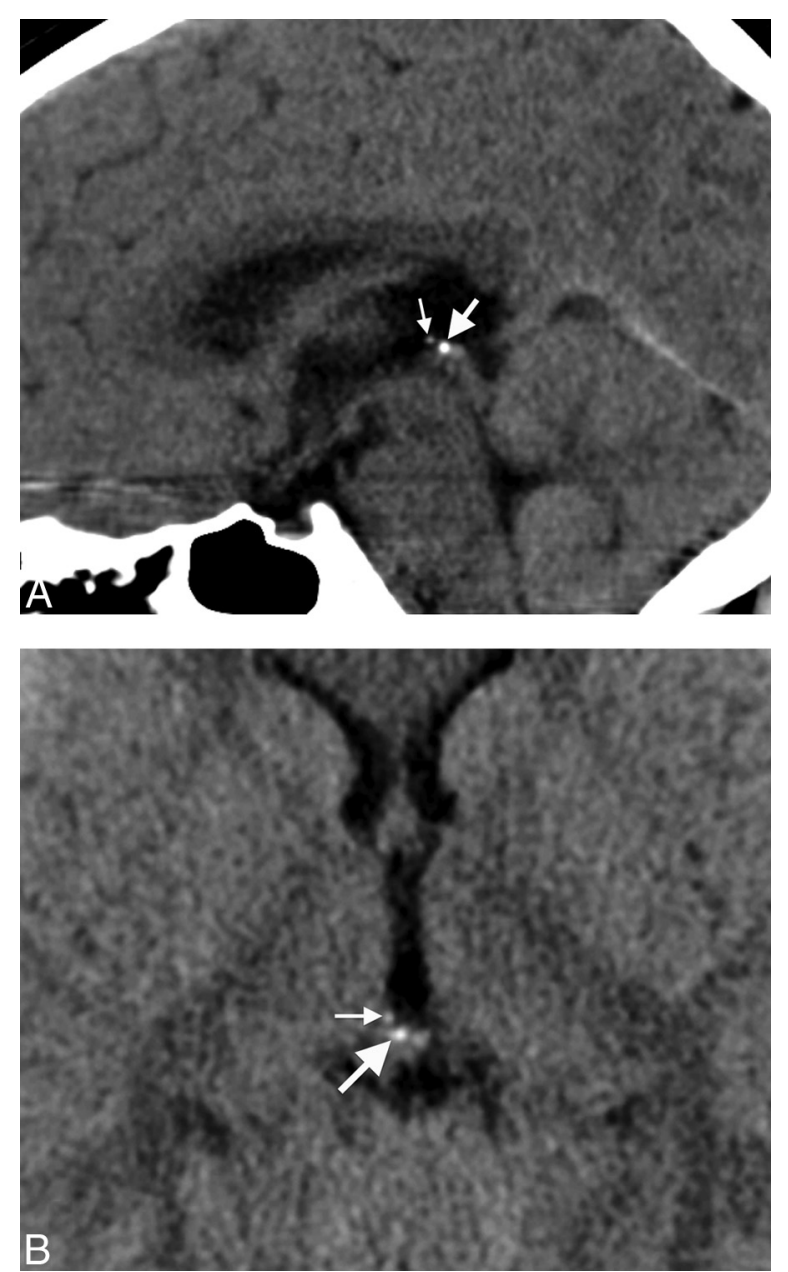

FIG 2. A, Midline sagittal head CT image from an 8-year-old child obtained for headache, demonstrating calcifications in both the pineal gland (large arrow) and habenular complex (small arrow). Habenular calcifications are centered anterosuperior and lateral to the pineal base. $B$, Axial head CT image through the epithalamus from an 8-year-old child performed for headache, showing pineal gland (large arrow) and habenular (small arrow) calcifications. Habenular calcifications are centered anterolateral to the pineal base.

\section{Choroid Plexus}

Twelve percent of subjects $(n=58)$ had choroid plexus calcifications (age range, $0.1-8.8$ years; median, 6 years; Fig 3 ). All choroid plexus calcifications were localized to the lateral ventricular glomus. Most choroid plexus calcifications were punctate and/or few $(n=54)$. The 4 remaining patients with large and/or numerous choroid plexus calcifications were $8(n=3)$ and $3(n=1)$ years of age. There was a significant association between patients with choroid plexus and pineal calcifications $(P=.003)$.

\section{Dura}

Dural calcifications were rare, present in only $1 \%(n=6$; age range, 2.9-8.7 years; median, 6 years; Fig 4 ). The tentorium was the most common dural site to calcify $(n=4)$ followed by the falx cerebri $(n=2)$.

\section{DISCUSSION}

\section{Epithalamus}

The epithalamus comprises the pineal gland and habenular complex. We chose to examine the prevalence of epithalamic, pineal,

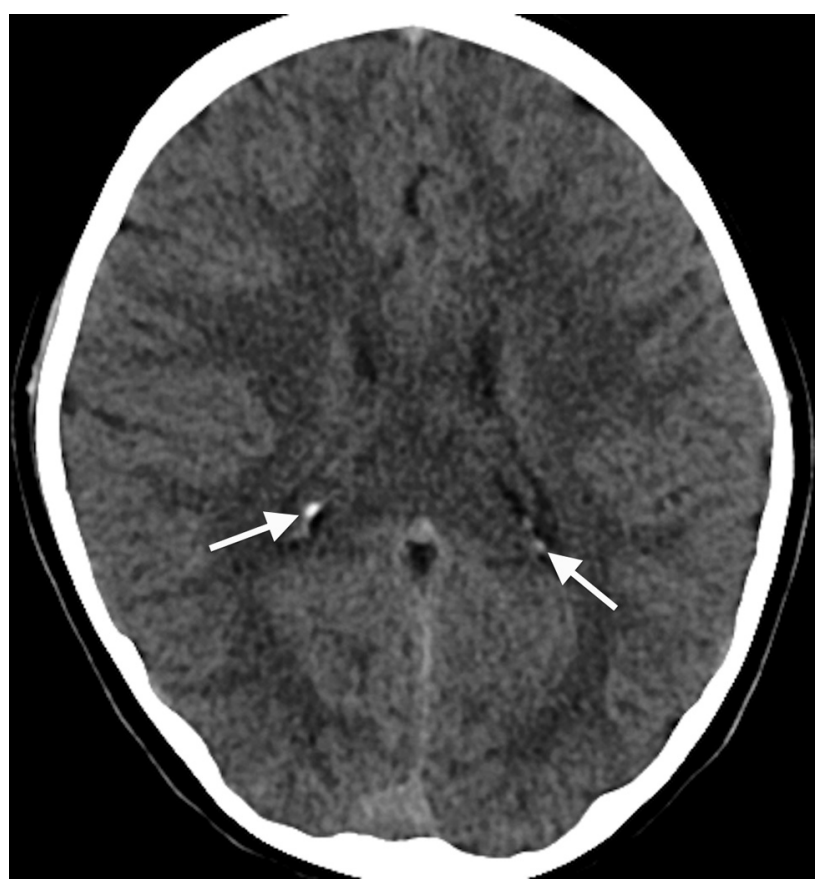

FIG 3. Axial head CT image from an 8-year-old child obtained for seizure, at the level of the lateral ventricular atria, depicting choroid plexus glomus calcifications (arrows).

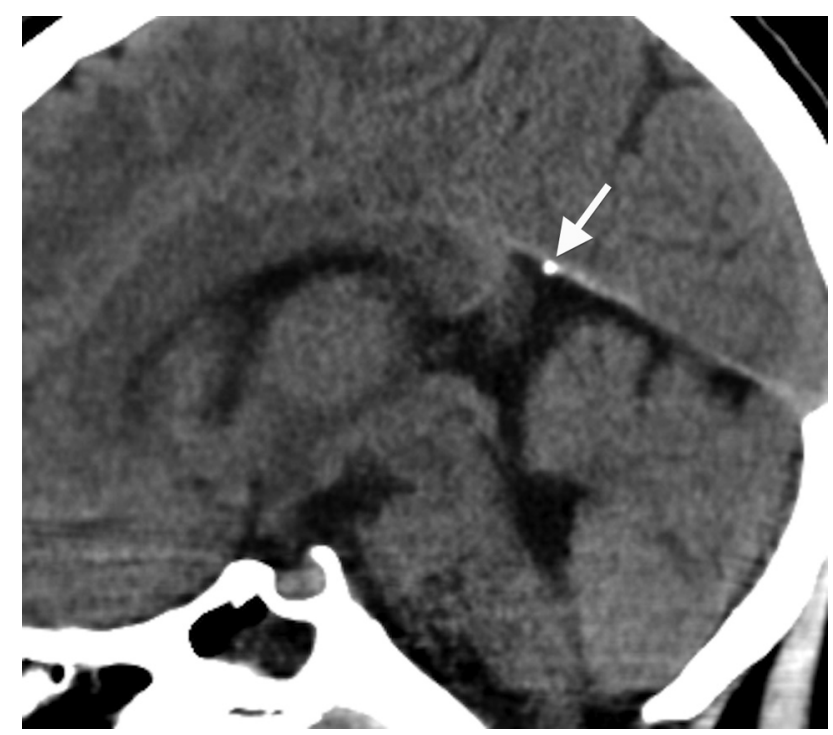

FIG 4. Midline sagittal head CT image from an 8-year-old child obtained for headache, demonstrating dural calcifications along the anterior margin of the cerebellar tentorium (arrow).

and habenular calcifications separately for several reasons. First, we think pineal and habenular calcifications can be accurately distinguished in most circumstances with modern CT technology and thin-section images. Second, we suspect that in the past, many habenular calcifications were misclassified as being pineal. Because these structures are functionally unique, we think that they deserve to be analyzed separately.

\section{Pineal Gland}

The pineal gland is a conical organ situated in the quadrigeminal cistern between the posterior and habenular commissures. Pi- 
nocytes, glial cells, neurons, and perivascular phagocytes constitute the bulk of its internal structure. ${ }^{4}$ After a period of initial growth, the size of the pineal gland has been shown to remain stable beyond the first 1-2 years of life. ${ }^{5-7}$ Knowledge of normal pineal gland size/volume is relevant in the evaluation of pineal region pathology. Pineal calcifications that would be considered benign in isolation are suspicious for neoplasia if there is concurrent glandular enlargement.

Why does the pineal gland calcify, and where is the calcium localized? Calcified concretions called "corpora arenacea" or "brain sand" found within the pineal gland primarily comprise calcium and magnesium salts. ${ }^{8}$ Exophytic membrane debris, a by-product of pineal neuronal and glial polypeptide exocytosis, forms the skeleton of the corpora arenacea around which calcifications are laid. ${ }^{9}$ Early calcifications found in younger patients are globular and localize near pinealocytes; whereas in older patients, calcifications tend to be more concentric and are associated with glial cells. ${ }^{10}$ In a study of older adults (age range, $62-80$ years), Kim et al ${ }^{11}$ found multiple concentrically calcified areas distributed throughout the pineal gland with centrally concentrated larger aggregates. Perivascular, tryptase-secreting mast cells have been found in areas where intrapineal calcium deposits originate, implicating these mast cells in the calcification process. ${ }^{1}$ Calcium also tends to localize along cell membranes. ${ }^{8}$ The outer pineal gland membrane may calcify, generally in a laminated manner. ${ }^{8,10}$ The laminated pineal calcifications that are found in older individuals may be related to circannual calcium level changes. ${ }^{8}$ However, the underlying cause of pineal calcium deposition remains elusive. It could represent a physiologic, maturational, or degenerative process.

In 1982, Zimmerman and Bilaniuk ${ }^{2}$ studied a large series of 725 patients (age range, $0-20$ years) to define the incidence of pineal calcifications in the first 2 decades of life. In that study, the incidence of physiologic pineal calcifications was $11 \% ; 13 \%$ of these were found in the first decade, with the youngest patient being 6.5 years. Because the study used CT parameters that would be considered primitive by today's standards, including a relatively small matrix $(160 \times 160$ or $320 \times 320)$ and large section thickness $(8 \mathrm{~mm})$, the incidence of pineal calcifications was likely underestimated. Other literature contradicts the low range of 6.5 years. One study indicated a 3\% frequency of pineal calcifications in the first 12 months. ${ }^{12}$ A more recent study by Doyle and Anderson $^{13}$ demonstrated pineal calcifications in $8 \%$ of patients $0-10$ years of age by using CT with a $512 \times 512$ matrix and $8-\mathrm{mm}$ section thickness in nearly all cases. The youngest patient with pineal calcification was 3 years of age, and $1 \%$ of patients younger than 6 years had pineal calcifications, identical to our study results. Early pineal glandular calcium deposition has also been shown in the histopathology literature, where microscopic calcifications in the human pineal gland have been documented even before birth. ${ }^{1}$

We found pineal calcifications in 5\%. While most of these patients were older than 5 years, a few were younger (youngest, 3 years). The apparent decrease in the percentage of patients with pineal region calcifications between 7 and 9 years of age is likely attributable to fewer patients in older patient subsets (Fig 1). The mantra that no pineal gland calcification should be visible in pa- tients younger than 6 years is no longer relevant in the modern CT age. ${ }^{2}$ However, pineal calcifications were single and punctate in all of our patients younger than 7 years of age. Occasional cases of larger and more numerous pineal calcifications were seen only in patients older than 7 years. Therefore, we advise cautious scrutiny in those patients younger than 7 years manifesting large and/or excessive pineal calcifications. Suspicious calcifications (premature, large, or excessive) should prompt a glandular volume analysis. ${ }^{5-7}$ A detailed clinical and biochemical assessment should be undertaken. Follow-up imaging should also be considered to exclude early presentation of a pineal tumor.

\section{Habenula}

The habenula is a small, bilaterally paired nuclear complex located along the inferior dorsomedial thalami. It is structurally connected to many deep brain structures, including the pineal gland, hypothalamus, midbrain, basal ganglia, and septal area, to name a few. Thus, it has a multitude of functions, mainly serving as a relay and processing center between the limbic system and midbrain through the stria medullaris thalami (afferent) and habenulointerpeduncular tract (efferent), ultimately influencing dopamine and serotonin elaboration. ${ }^{14}$ The habenular complex also participates in the sleep-wake cycle. The retinohypothalamic reflex is the process by which light-induced retinal activation recognized in the hypothalamus signals the pineal gland to decrease melatonin production by way of the sympathetic chain. One function of melatonin is to inhibit the habenular complex. In the absence of melatonin-induced habenular inhibition, dopamine inhibition, normally mediated by the habenula, is eliminated, and dopamine excretion increases. ${ }^{14,15}$ Because the pineal gland and habenula are in proximity and share at least 1 common function, it is understandable that they may calcify at similar times, whether degenerative, physiologic, or otherwise. The substance of these calcifications is similar in makeup to that found in the pineal gland; the calcifications contain both calcium and magnesium salts. ${ }^{8}$

To our knowledge, the potential association between sleep dysfunction and habenular calcification has not been systematically examined. However, there may be a link between habenular calcification and pathophysiology, ultimately disrupting the aversion/reward process. For instance, pineal and habenular calcifications have been noted as frequent findings in patients with schizophrenia. ${ }^{16,17}$ Because patients with schizophrenia often have difficulty learning from errors, habenular dysfunction has been implicated. ${ }^{18}$ Habenular calcifications were the most common epithalamic calcification in our cohort, present in $10 \%$ and in none younger than 2 years of age.

\section{Choroid Plexus}

The choroid plexus is an intraventricular organ comprising cuboidal epithelial cells, vasculature, and connective tissue. It has multifaceted functionality, chiefly serving in CSF volume modulation with productive, absorptive, and filtering capability. Its bulk resides in the lateral ventricular atria, called the glomus. During the human life span, the choroid plexus tends to accumulate physiologic calcifications. Because most choroid plexus is concentrated in the ventricular atria, it is here that calcifications are 
most frequently evident. Pathologic processes such as prior intraventricular infection, inflammation, and hemorrhage and chronic calcium and phosphate imbalance can cause premature or excessive choroid plexus mineralization. Therefore, knowledge of a normally expected calcification time range would be helpful in distinguishing physiology from pathology.

The prevalence of choroid plexus calcification increases with age. ${ }^{19,20}$ However, few studies have examined choroid plexus calcifications in children. In a large retrospective CT study from 18,000 consecutive children ( $0-15$ years of age) performed with early CT technology (EMI 1010 CT) by Kendall and Cavanagh, ${ }^{20}$ choroid calcifications were found in $2 \%$ of patients younger than 8 years of age. Another CT study that examined scans obtained with 8 -mm section thickness and a $256 \times 256$ matrix demonstrated the prevalence of choroid plexus calcification in the first decade to be $0.5 \% .^{3}$ More recently, Doyle and Anderson ${ }^{13}$ found choroid plexus calcifications to be more prevalent, present in 16\% of their patients. Indeed, choroid plexus psammoma bodies have been described in children histopathologically. ${ }^{21}$ Dystrophic calcification associated with the arachnoid cells and collagen fibers is thought to be causative. ${ }^{22}$

We discovered choroid plexus calcifications in $12 \%$ of patients in our cohort. In accordance with findings described by Doyle and Anderson, ${ }^{13}$ we found a significant positive association of choroid plexus and pineal calcifications. Choroid calcifications were visible in patients across all age ranges, from younger than 1 month to 10 years. A variety of clinical scenarios brought these patients to imaging, mainly trauma and headache. In all cases, the choroid plexus glomus was calcified in isolation, either unilaterally or bilaterally. Typically, calcifications were punctate and few. Therefore, choroid plexus calcifications occurring outside the glomus or in excess should be viewed with suspicion for underlying pathology in patients younger than 9 years of age. Minimal choroid plexus calcium deposition is probably physiologic at any age, including early childhood.

\section{Dura}

Thick connective tissue composing the dura envelops the central nervous system deep to the skeleton. Dura partitions the intracranial compartment into various subsections, forms the walls of the major dural venous sinuses, and serves as a conduit for neurovascular connections.

Although extremely rare, the CT prevalence of childhood dural calcifications remains a subject without strong support in the literature. In a series of 970 trauma patients, Ratcliffe et $\mathrm{al}^{23}$ found dural calcifications in $>20 \%$ of men older than 30 years and women older than 50 years on skull radiography; attenuated calcifications were present in less than $2 \%$ of those younger than 40 years. Kendall and Cavanagh ${ }^{20}$ found dural calcifications in $0.8 \%$ of patients $0-15$ years of age. Dural calcifications were rare in our study, occurring in $1 \%$. Posttraumatic dural calcifications rarely occur but are not unexpected after a calvarial breach from penetrating trauma and surgical intervention. Indeed, most of our patients with dural calcifications had previous craniotomies $(4 / 6)$. The other 2 presented with headache ( 9 years of age) and head trauma (4 years of age). The tentorium was the most common area to calcify. Extensive dural calcifications in young patients should raise suspicion for pathologic conditions, including basal cell nevus syndrome, meningiomas, sequelae of prior subdural or epidural hemorrhage, and calcium/phosphate imbalance. ${ }^{24}$

This study has several limitations. Calcium detection is inherently limited by CT section thickness; therefore, intracranial calcifications may have been underestimated. However, we believe this underestimation to be of little clinical importance because thinner section soft-tissue algorithm images are not currently part of most practices. Despite their distinctive locations, pineal and habenular calcifications can sometimes be difficult to separate visually; this problem could have resulted in misclassification in some instances (Fig 2). Pineal cysts are common in the age group in our study and can distort regional anatomy. ${ }^{7}$ We found pineal cysts in $3 \%$ of our patient cohort, ranging from 4 to $15 \mathrm{~mm}$; however, only 4 of these patients had epithalamic calcifications.

\section{CONCLUSIONS}

Physiologic intracranial calcifications may be found in the first decade, principally in children older than 5 years. Most epithalamic calcifications are habenular in location. Pineal and habenular calcifications were never present in children younger than 3 and 2 years, respectively. Dural calcifications are rare; they are not present in those younger than 2 years of age. Choroid plexus calcifications may be present in the very young (younger than 1 year).

\section{REFERENCES}

1. Maślińska D, Laure-Kamionowska M, Deręgowski K, et al. Association of mast cells with calcification in the human pineal gland. Folia Neuropathol 2010;48:276-82

2. Zimmerman RA, Bilaniuk LT. Age-related incidence of pineal calcification detected by computed tomography. Radiology 1982;142:659-62

3. Modic MT, Weinstein MA, Rothner AD, et al. Calcification of the choroid plexus visualized by computed tomography. Radiology 1980;135:369-72

4. Nieuwenhuys R, Voogd J, van Huijzen C. The Human Central Nervous System. 4th ed. Berlin: Springer-Verlag; 2008:247-50

5. Schmidt F, Penka B, Trauner M, et al. Lack of pineal growth during childhood. J Clin Endocrinol Metab 1995;80:1221-25

6. Sumida M, Barkovich J, Newton TH. Development of the pineal gland: measurement with MR. AJNR Am J Neuroradiol 1996; 17:233-36

7. Whitehead MT, Oh C, Choudhri AF. Incidental pineal cysts in children who undergo 3-T MRI. Pediatr Radiol 2013;43:1577-83

8. Vigh B, Szel A, Debreceni K, et al. Comparative histology of the pineal gland. Hisol Histopathol 1998;13:851-70

9. Lukaszyk A, Rieter RJ. Histopathological evidence for the secretion of polypeptides by the pineal gland. Am J Anat 1975;143:451-64

10. Koshy S, Vettivel SK. Varying appearance of calcification in human pineal gland: a light microscopic study. J Anat Soc India 2001;50:17-18

11. Kim J, Kim HW, Chang $S$, et al. Growth patterns for acervuli in human pineal gland. Sci Rep 2012;2:984

12. Helmke K, Winkler P. Incidence of pineal calcification in the first $\mathbf{1 8}$ years of life [in German]. Rofo 1986;144:221-26

13. Doyle AJ, Anderson GD. Physiologic calcification of the pineal gland in children on computed tomography: prevalence, observer reliability and association with choroid plexus calcification. Acad Radiol 2006;13:822-26

14. Bianco I, Wilson SW. The habenular nuclei: a conserved asymmetric relay station in the vertebrate brain. Philos Trans $R$ Soc Lond $B$ Biol Sci 2009;364:1005-20 
15. Kunz D, Schmitz S, Mahlberg R, et al. A new concept for melatonin deficit: on pineal calcification and melatonin excretion. Neuropsychopharmacology 1999;21:765-72

16. Sandyk R. Pineal and habenular calcification in schizophrenia. Int J Neurosci 1992;67:19-30

17. Caputo A, Chiringheli L, Dieci M, et al. Epithalamus calcifications in schizophrenia. Eur Arch Psychiatry Clin Neurosci 1998;248:272-76

18. Shepard PD, Holcomb HH, Gold JM. Schizophrenia in translations: the presence of absence: habenular regulation of dopamine neurons and the encoding of negative outcomes. Schizophr Bull 2006;32:417-21

19. Admassie D, Mekonnen A. Incidence of normal pineal and chroids plexus calcification on brain CT (computerized tomography) at Tikur Anbessa Teaching Hospital Addis Ababa, Ethiopia. Ethiop Med J 2009;47:55-60
20. Kendall B, Cavanagh N. Intracranial calcification in paediatric computed tomography. Neuroradiology 1986;28:324-30

21. Korzhevskii DE. The formation of psammoma bodies in the choroid plexus of the human brain. Morfologiia 1997;111:46-49

22. Alcolado JC, Moore IE, Weller RO. Calcification in the human choroid plexus, menigiomas and pineal gland. Neuropathol Appl Neurobiol 1986;12:235-50

23. Ratcliffe JF, Shanley S, Ferguson J, et al. The diagnostic implication of falcine calcification on plain skull radiographs of patients with basal cell naevus syndrome and the incidence of falcine calcification in their relatives and two control groups. $\mathrm{Br} J$ Radiol 1995; 68:361-68

24. Makariou E, Patsalides AD. Intracranial calcifications. Appl Radiol 2009;38:48-60 